\title{
Radiotherapy induces responsiveness of a resistant mammary carcinoma to PD-1 blockade
}

\author{
Julie Diamond*, Karsten Pilones, Joseph Aryankalayil, Ralph Vatner, Silvia Formenti, Sandra Demaria \\ From Society for Immunotherapy of Cancer 29th Annual Meeting \\ National Harbor, MD, USA. 6-9 November 2014
}

\begin{abstract}
Background
We've previously shown that radiotherapy (RT) converts poorly immunogenic tumors resistant to antibodies (mAbs) against the immune checkpoint receptor CTLA-4 into susceptible ones (Demaria et al., 2005; Dewan et al., 2009). Programmed death-1 (PD-1) is another immune checkpoint receptor upregulated on T-cells shortly after activation and expressed at high levels on exhausted Tcells. Anti-PD-1 mAbs have shown marked clinical activity in some cancer patients, but the majority does not respond. Here we tested the hypothesis that RT can sensitize poorly immunogenic tumors to anti-PD-1.
\end{abstract}

\section{Methods}

$\mathrm{BALB} / \mathrm{c}$ mice were subcutaneously inoculated with syngeneic, poorly immunogenic TSA breast carcinoma cells on day0. When tumors became palpable, mice were randomly assigned to one of four treatment groups: control, RT, anti-PD-1 and RT+anti-PD-1. Local RT was administered to the tumor in three 8Gy fractions on days 13,14 , 15. PD-1-blocking mAb RMP1-14 was given on day15 and every 4 days thereafter. Mice were followed for tumor growth. In a separate experiment, mice were euthanized on day 20 to characterize tumor-infiltrating lymphocytes (TILs) and development of CD8 ${ }^{+} \mathrm{T}$ cells specific for tumor epitope, AH1, using pentamer analysis.

\section{Results}

Expression of activation markers CD69 and CD137 was increased in $\mathrm{CD}^{+}$TILs from mice treated with $\mathrm{RT}$, while RMP1-14 was ineffective (64\%-RT or $70 \%$-RT +RMP1-14 compared to 42\%-control and 47\%-RMP1$14, \mathrm{p}<0.001)$. RT-treated mice also showed significant increase in $\mathrm{CD}^{+}$TILs expressing high levels of PD-1 $\left(\mathrm{CD}^{+} \mathrm{PD}-1^{\mathrm{hi}}\right)(67 \%$ vs $36 \%, p<0.01)$. Importantly, PD-1 ligands, PDL-1/2, were upregulated by RT on TSA cells and tumor-infiltrating myeloid cells, suggesting PD-1's interaction with its ligands may limit RT-activated antitumor T-cell activity. Consistent with this hypothesis, RMP1-14 alone had no effect on tumor growth; RT delayed growth $(\mathrm{p}<0.01)$, but only $1 / 6$ mice showed tumor regression, whereas all mice receiving RT+RMP114 completely rejected tumors by day 25 . In spleen, RMP1-14 had no effect on AH1-specific CD8 ${ }^{+}$T-cells (1.8\% vs $1.7 \%$-control) while RT significantly expanded this population $(2.9 \%, \mathrm{p}<0.05)$. RT + RMPI-14, however, demonstrated the highest increase $(4.6 \%, \mathrm{p}<0.05$ vs. all other groups).

\section{Conclusion}

These results suggest dual benefits of anti-PD-1 when used in combination with RT. PD-1 blockade enhances $\mathrm{RT}$-induced $\mathrm{T}$ cell priming, possibly by decreasing the TCR activation threshold. Simultaneously, anti-PD-1 recovers $\mathrm{T}$-cell effector activity in the tumor by abrogating the inhibitory signals mediated by RT-induced PD-1 ligands. Data strongly supports testing this combination in the clinic.

Supported by the Breast Cancer Alliance (Exceptional Project Award).

Published: 6 November 2014

doi:10.1186/2051-1426-2-S3-P159

Cite this article as: Diamond et al: Radiotherapy induces responsiveness of a resistant mammary carcinoma to PD-1 blockade. Journal for

ImmunoTherapy of Cancer 2014 2(Suppl 3):P159.

NYU School of Medicine, New York, NY, USA 